\title{
Selección fenotípica de árboles plus de tres especies forestales maderables en poblaciones naturales en el
} Distrito de Molinopampa (Amazonas)

\section{Fenotypic selection of trees plus three wood forest species in natural populations in the District of Molinopampa (Amazonas)}

\author{
Mario Oliva $^{1^{*}}$ e Ysaías Rimachi
}

\section{RESUMEN}

El estudio se realizó en el distrito de Molinopampa en el departamento de Amazonas, cuyo objetivo fue seleccionar Árboles Plus de las especies maderables de "aliso" Alnus acuminata, "cedro" Cedrela odorata e "ishpingo" Ocotea $s p$. El trabajo se inició identificando árboles candidatos plus en el ámbito del bosque de palmeras, estos árboles fueron evaluados en sus características dasométricas, sanidad en base a sus vecinos, a este nivel logrando una primera discriminación de 108 árboles candidatos plus distribuidos en 42 árboles de aliso, 30 árboles de cedro y 36 árboles de ishpingo. Los árboles candidatos plus fueron sometidos a pruebas de producción de brotes en fuste. Los ensayos fueron montados de manera independiente para cada especie bajo DBCA con arreglo factorial 2Ax3B teniendo como Factor A: diámetro a la altura del pecho y Factor B: tipo de corte en fuste. Se evaluo el periodo de producción de brotes y rendimiento productivo por árbol. Los datos fueron analizados, encontrando una mayor respuesta en producción de brotes en árboles con DAP menor a $30 \mathrm{~cm}$ y corte transversal. En base a esto logrando la selección definitiva de 23 Árboles Plus de aliso, 18 Árboles Plus de cedro y 20 Árboles Plus de ishpingo.

Palabras claves: rodal, árboles candidatos, fenología, rebrotes.

\section{ABSTRACT}

The study was carried out in the district of Molinopampa in the department of Amazonas, whose objective was to select plus trees of the woody species of "alder" Alnus acuminata, "cedar" Cedrela odorata and "ishpingo" Ocotea sp. The work began by identifying plus candidate trees in the field of palm forest, these trees were evaluated in their dasometric characteristics, sanitation based on their neighbors, at this level achieving a first discrimination of 108 candidate trees plus distributed in 42 alder trees , 30 cedar trees and 36 ishpingo trees. The plus candidate trees were tested for stem shoot production. The assays were assembled independently for each species under DBCA with factorial arrangement 2Ax3B having as Factor A: diameter at breast height and Factor B: type of cut at bole. The period of production of shoots and productive yield per tree was evaluated. The data were analyzed, finding a greater response in shoot production in trees with DBH less than $30 \mathrm{~cm}$ and cross section. Based on this achieving the final selection of 23 trees plus alder, 18 trees plus cedar and 20 trees plus ishpingo.

Key words: rodal, candidate trees, phenology, regrowths.

\footnotetext{
${ }^{1}$ Asociación para la Conservación del Bosque de Palmeras. Jr. Santo Domingo Nº64-C. Chachapoyas. Amazonas. Perú.

"Autor de correspondencia. E-mail: agroliva.123@hotmail.com.
} 


\section{INTRODUCCIÓN}

Los materiales forestales de reproducción como frutos, semillas, plantas y partes de plantas utilizadas en el proceso de germinación natural o artificial, constituyen parte esencial de todo proceso de reforestación de bosques; además, a corto plazo, son elementos básicos para realizar repoblamiento, y sus particulares morfológicas y genéticas determinan las características de las futuras masas forestales en términos de composición, adaptación y crecimiento, de tal modo que, el manejo silvicultural de los rodales naturales se orienta a obtener la mayor cantidad de semillas con la calidad deseada (Gutiérrez et al., 2003).

La identificación y selección del árbol de alto rendimiento, es el inicio y la base fundamental de un programa de mejoramiento genético forestal. En relación con la calidad y rigurosidad con que se realice la selección de estos árboles, así será en concordancia la ganancia genética que se alcanzará. La rigurosidad en la selección se estima a través del concepto conocido como intensidad de selección "i”,; y se puede también expresar en términos de la magnitud del diferencial de selección "S", que se define de manera clásica como la distancia entre el promedio del conjunto de los individuos seleccionados y el promedio de la población original, para un carácter determinado (Chang, 2007).

Los árboles se seleccionan separados al menos por 100 $\mathrm{m}$ de distancia uno de otro, a fin de establecer posteriormente ensayos de progenie, donde sí se podrá, entonces en condiciones de plantación, realizar una selección eficiente y certera (Murillo et al., 2010). Sin embargo, aún cuando se logre una selección estricta del material, poco se avanzará en el trabajo de mejoramiento si no hay una alta variabilidad genética en la población para el o los caracteres a mejorar. Debe recordarse que la selección de los árboles plus se realiza exclusivamente en plantaciones, con base en la expresión fenotípica de caracteres de interés. Por tanto, la manifestación de estos caracteres está siempre bajo efectos ambientales y, efectos genéticos, que podrían ser muy altos en algunos casos y confundir al seleccionador con lo que observe fenotípicamente (Jara, 1994).

Los árboles semilleros deben contar con la edad suficientemente joven para poder desarrollar copas frondosas y una buena producción de semillas; asimismo, la calidad genética de la fuente de semilla de especies arbóreas es el factor decisivo de los buenos resultados para cualquier programa de reforestación. La selección de una fuente de semilla parte del supuesto, es muy probable, de que las características de los árboles de los cuales se cosechan las semillas sean transmitidas a su descendencia (Sotelo y Weber, 1997). El objetivo de la presente investigación es seleccionar Árboles Plus de las especies forestales maderables de aliso, cedro e ishpingo.

\section{MATERIALES Y MÉTODOS}

\section{Localización del estudio}

El estudio se realizó en el distrito de Molinopampa, provincia de Chachapoyas, región Amazonas, cuyas coordenadas geográficas corresponden a $18 \mathrm{M}$ 0216327 UTM 9306478, a una altitud de $2250 \mathrm{msnm}$. El ámbito del estudio le se corresponde con la zona de vida del bosque de palmeras (género Ceroxylon), que presenta una temperatura media de $15^{\circ} \mathrm{C}$ y una precipitación promedio anual de $1800 \mathrm{~mm}$, con cinco5 meses de época seca y una humedad relativa del 76.06\%. Los límites colindantes del distrito de Molinopampa son los siguientes: por el norte limita con los distritos de Quinjalca y Granada, por el sureste con la provincia de Rodríguez de Mendoza, por el suroeste con el distrito de Cheto y por el oeste con los distritos de San Francisco de Daguas y Sonche (Figura 1).

\section{Identificación de árboles candidatos plus}

La selección de Árboles Plus, se realizó en un ámbito de aproximadamente 460 hectáreas distribuidos en tres anexos del distrito de Molinopampa, interviniendo en al menos cinco5 rodales (Rodal Patigallo 90 ha, 


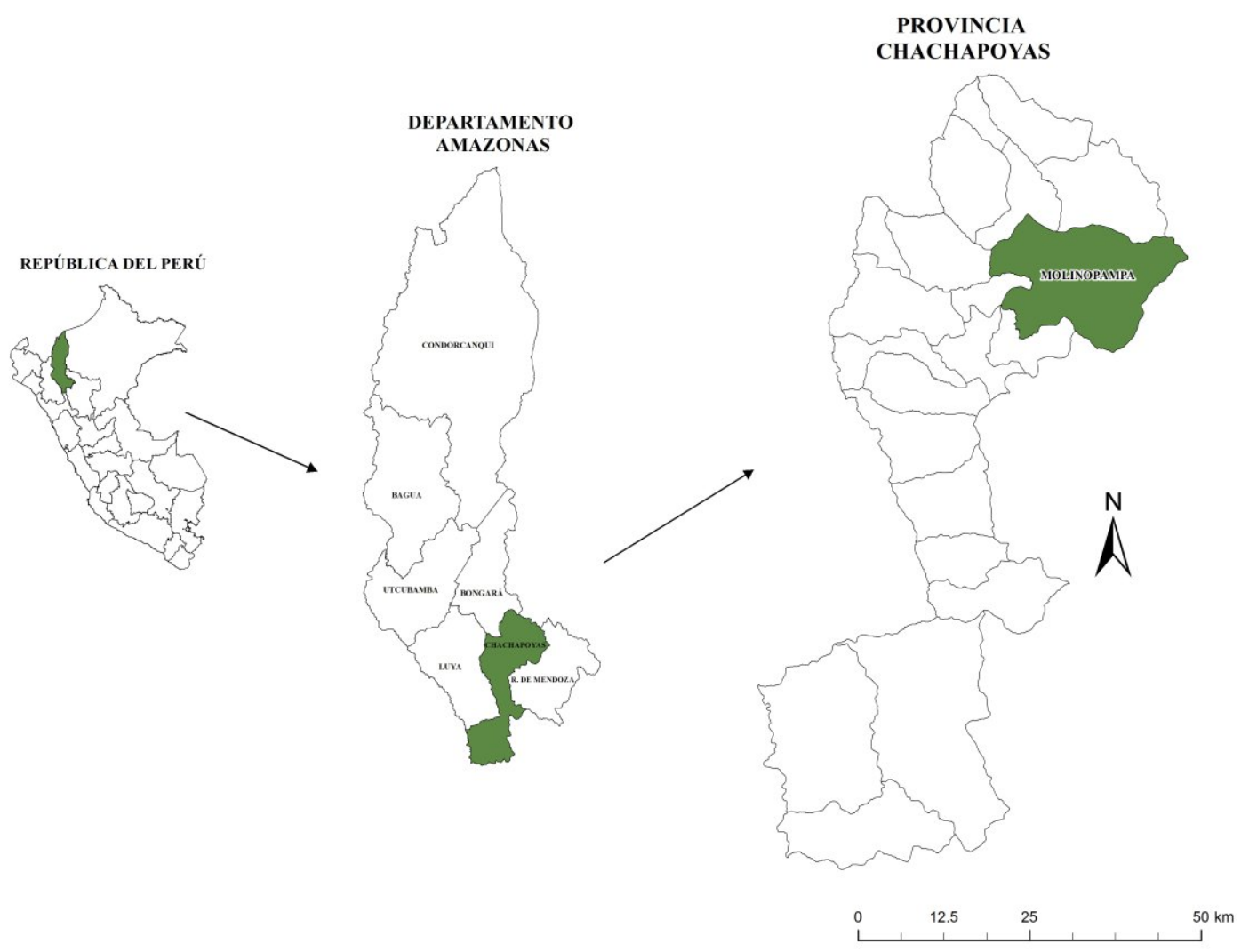

Figura 1. Ubicación geográfica del área de estudio en el distrito de Molinopampa, provincia de Chachapoyas (departamento de Amazonas)

Rodal Allpachaca 95 ha, Rodal Ocol 85 ha, Rodal Mishquiaco 92 ha y Rodal Tragadero 98 ha). Para desarrollar la preselección de Árboles Plus se utilizó la técnica de "selección específica" o "método de valoración individual" logrando identificar 42 árboles de aliso (Alnus acuminata), 30 árboles de cedro (Cedrela sp.) y 36 árboles de ishpingo (Nectandra sp.)..

En la selección de los árboles candidatos se consideraron criterios establecidos previamente, los cuales se definieron con base en su heredabilidad (carácter fenotípico) y su fácil medición (carácter dasométrico). Los caracteres más representativos fueron: la manifestación genética (DAP) y el estado fitosanitario (el cual se expresa en el periodo juvenil y repercute en el periodo adulto). Los caracteres que se aplicaron en el mejoramiento fueron: diámetro a la altura del pecho entre 20 a $40 \mathrm{~cm}$, altura total entre 8 a $20 \mathrm{~m}$, estado fitosanitario y calidad del fuste. Para la selección de los árbo- les candidatos plus se utilizó un GPS Garmin Oregon 650, motosierra mediana Sthil 381, machetes, serrucho cola de zorro y un formón de 1 pulgada. Finalmente cada árbol candidato plus con un DAP y altura total dentro del rango establecido fue codificado y marcado en el fuste utilizando pintura.

\section{Manejo silvicultural de árboles identificados}

Fertilización. La fertilización de árboles plus se realizó aplicando 200 gramos de fertilizante combinado de NPK (20-20-20) a un metro alrededor del árbol. Esta práctica permitió que el árbol disponga de mejores condiciones de fertilidad para solventar las necesidades de generación de biomasa en términos de rebrotes. Podas. Se realizaron podas de los árboles, ya que es importante en el funcionamiento de los vegetales por la relación que existe entre el crecimiento de las raíces y ramas. Las raíces principales de los árboles favorecen un mayor fluido de los nutrientes y hormonas 
naturales del suelo, importantes para la aparición de rebrotes.

Aclareo. Se realizó el aclareo mediante corte de árboles cercanos al candidato, además se se cortaron lianas y arbustos cercanos para permitir mayor entrada de luz solar. Si el árbol se ubica en bosque primario, el proceso de fotosíntesis se reliza con mayor facilidad, no obstante también es importante considerar un margen de sombra moderado para promover la elongación del rebrote.

Limpieza. La limpieza es importante puesto que la presencia de malezas atraerán insectos o grillos cortadores que pueden dañar los rebrotes. Por otra parte, la limpieza del árbol facilita ubicarlo desde lejos, permitiendo observar la placa de codificación del árbol aún en tiempos nublados.

Sanidad general. Se aplicó insecticida en polvo a razón de 50 gramos alrededor de cada árbol y en el tallo en la parte a inducir el brote, se observó la aparición de algunos insectos perforadores como pulverizadores de madera y termites. Es importante que el árbol este sano para facilitar el normal desarrollo de rebrotamiento.

Aplicación de citoquinina. Se aplicó citoquininas al $0.04 \%$ en el suelo cerca de la base del árbol con el fin de incrementar el desarrollo de brotes laterales, dado que la citoquinina promueve la elongación celular.

\section{Inducción de brotes en fuste}

Edad del árbol. Se evidenció que la parte basal del árbol es fisiológicamente más juvenil y activa que la parte superior del mismo, la productividad de los rebrotes está en función inversa a la edad del árbol, es decir los árboles adultos tienen la desventaja que el floema externo o súber esta cada vez más alejado de la corteza interna.

Dureza de la madera. Las especies forestales con densidad media y baja como el aliso y cedro fueron más propensos a la emisión de rebrotes basales, caso inverso sucedió con la especie ishpingo, lo cual resultó más dificultoso de rebrotar, atribuyéndose principalmente a la dureza de la madera.

Forma del corte. Se comparó 3 tipos de corte en fuste en árboles (transversal, forma de "V" y "V" invertida) bajo 3 repeticiones en al menos 18 árboles por cada especie maderable estudiada.

Altura del corte. La altura del corte en fuste fue estandarizado y se realizó a $50 \mathrm{~cm}$ medido desde la base de los árboles conla ayuda de una wincha y cinta diamétrica para medir el espesor del árbol.

Dimensiones del corte. Se realizó cortes con longitud no menor a un tercio, ni mayor a la mitad de la circunferencia para la forma transversal, con una profundidad de corte de $2 \mathrm{~cm}$, largo de $10 \mathrm{~cm}$ y de $3 \mathrm{~cm}$ de ancho.

Evaluación del crecimiento y desarrollo de los brotes

Se registraron los datos de desarrollo referidos al número de rebrotes, longitud del rebrotes, número de foliolos, diámetro de rebrote y tiempo promedio que tarda en producir el rebrote. Estos datos fueron anotados en formatos de evaluación por cada árbol intervenido de las tres especies estudiadas. El monitoreo se realizó cada 10 días y a partir de 72 días se realizó el conteo de brotes ya conformados con características requeridas para el proceso de enraizamiento.

Análisis del rendimiento de brotes y selección de árboles plus

Se consideró como criterio de selección de árboles plus el rendimiento promedio de brotes juveniles por árbol por cada especie forestal, seleccionándose aquellos árboles cuyo rendimiento es igual o mayor al promedio. Los datos fueron analizados mediante pruebas no paramétricas (distribuciones Chi-cuadrado): prueba de distribución multinomial y prueba de independencia, al nivel de confiabilidad de 95\%. Para el análisis de los datos se utilizó el programa estadístico SPSS versión 23 y la prueba de comparación del rango múltiple de Tukey $(0,05)$.

\section{RESULTADOS}

\section{Periodo de producción de brotes juveniles}

En la figura 2 se observa que el tipo de corte transversal aplicado en fuste de árboles de aliso con DAP entre 20 a $30 \mathrm{~cm}$, logró menor tiempo para la obtención de 
brotes juveniles, en promedio alcanzó 72,74 días desde el inicio de la inducción.

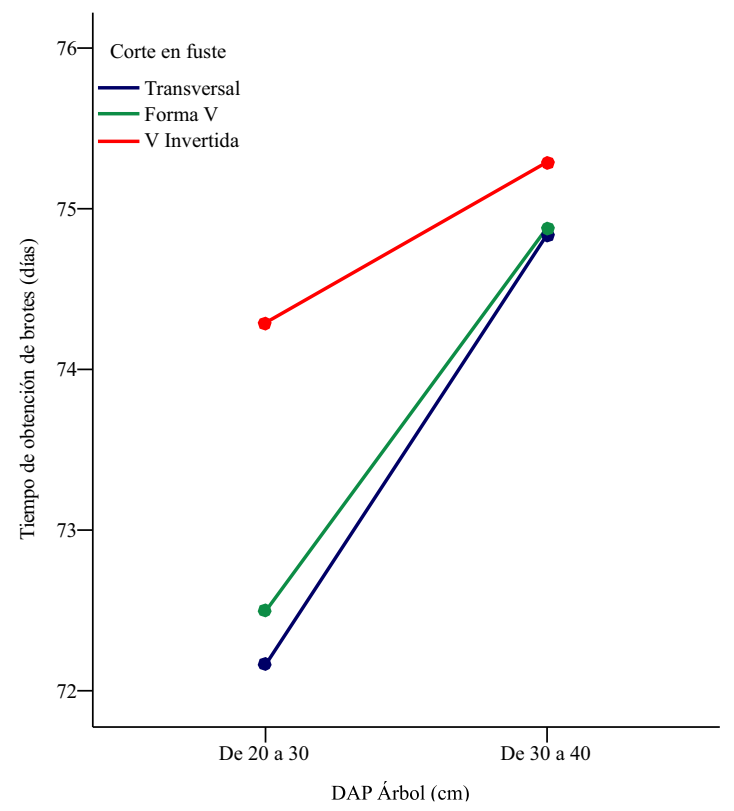

Figura 2. Periodo de obtención de brotes en árboles de aliso.

En tanto, los ensayos realizados en árboles de cedro evidenciaron que aplicando corte transversal en fuste en árboles con DAP entre 20 a $30 \mathrm{~cm}$ encontraron menor tiempo para la obtención de brotes juveniles, alcanzando en promedio 84,55 días, tal como se muestra en la figura 3.

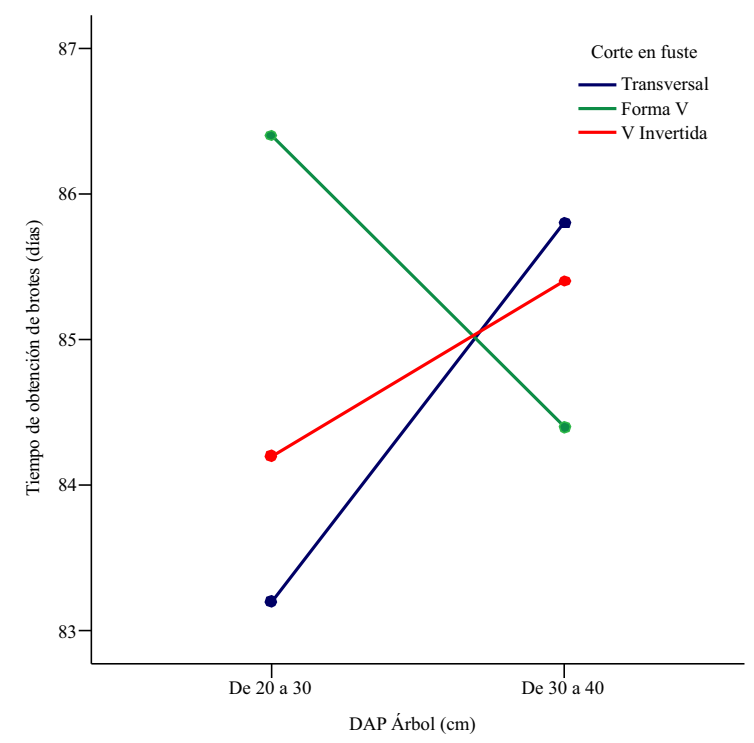

Figura 3. Periodo de obtención de brotes en árboles de cedro.
Ensayos realizados en árboles de ishpingo mostraron que aplicando corte en forma transversal en árboles con DAP entre 20 a $30 \mathrm{~cm}$, se obtuvieron en promedio 92,48 días para la obtención de brotes, lo cual fue muy semejante a ensayos aplicando corte en forma de $\mathrm{V}$ en árboles con DAP entre 20 a $30 \mathrm{~cm}$ (Figura 4).

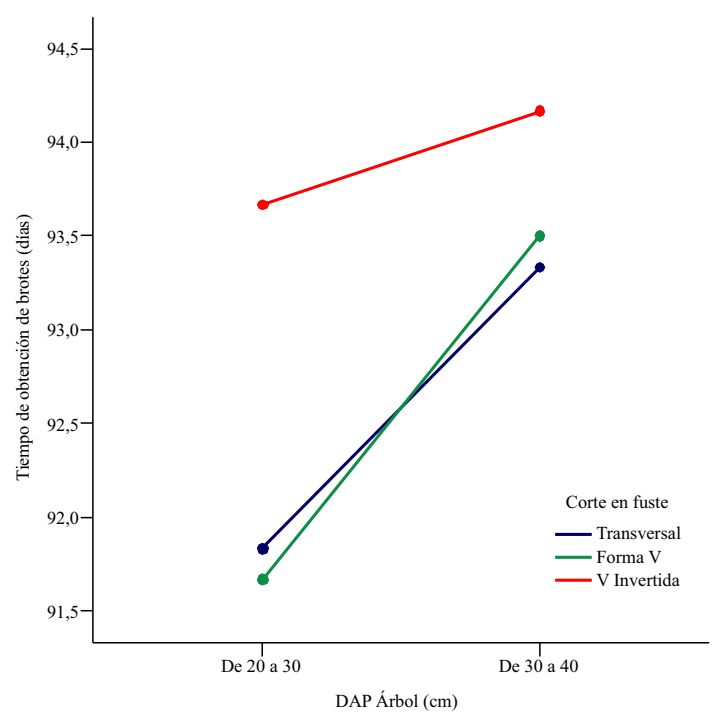

Figura 4. Periodo de obtención de brotes en árboles de ishpingo

\section{Rendimiento en la producción de brotes en árboles}

Respecto al rendimiento en producción de brotes juveniles en árboles de aliso se encontró mayor respuesta en árboles aplicados corte transversal y con DAP entre 20 a $30 \mathrm{~cm}$, en promedio alcanzó una producción de 3,60 brotes fértiles por árbol..

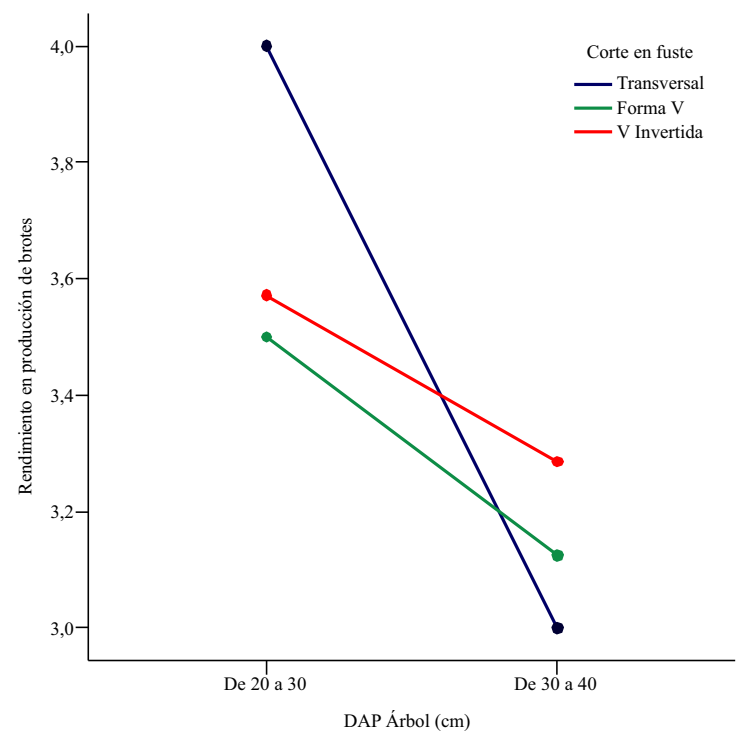

Figura 5. Rendimiento en producción de brotes de aliso. 
Para la especie cedro un mayor rendimiento productivo de brotes fue al orden de 2.62 brotes por árbol, cuyo rendimiento fue logrado en árboles aplicando corte transversal y con un DAP entre 20 a 30 cmap, tal como se aprecia en la figura 6.

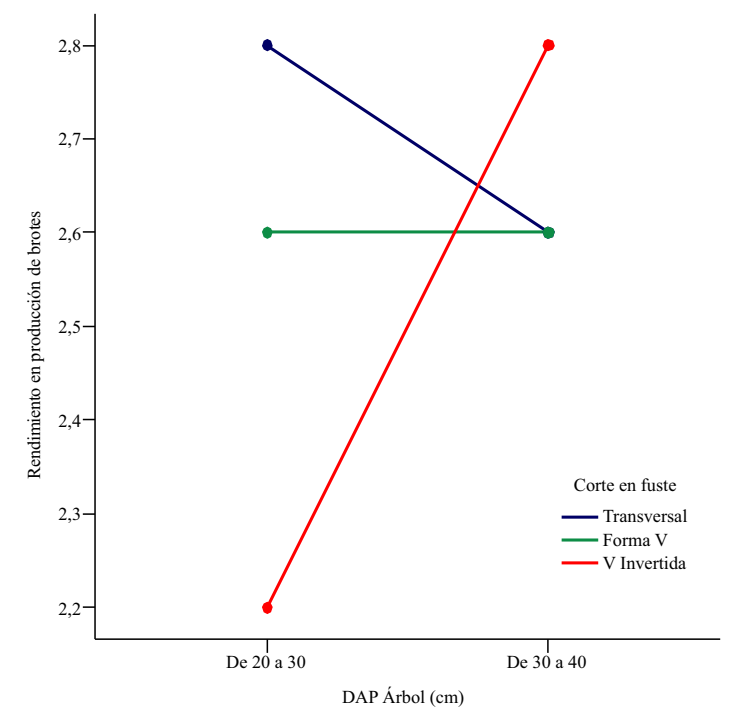

Figura 6. Rendimiento en producción de brotes de cedro.

La evaluación sobre el rendimiento productivo de brotes realizada en la especie ishpingo determinaron que aplicando corte transversal en árboles con DAP entre 20 a $30 \mathrm{~cm}$, alcanzaron en promedio 2.90 brotes por árbol, según se muestra en la figura 7.

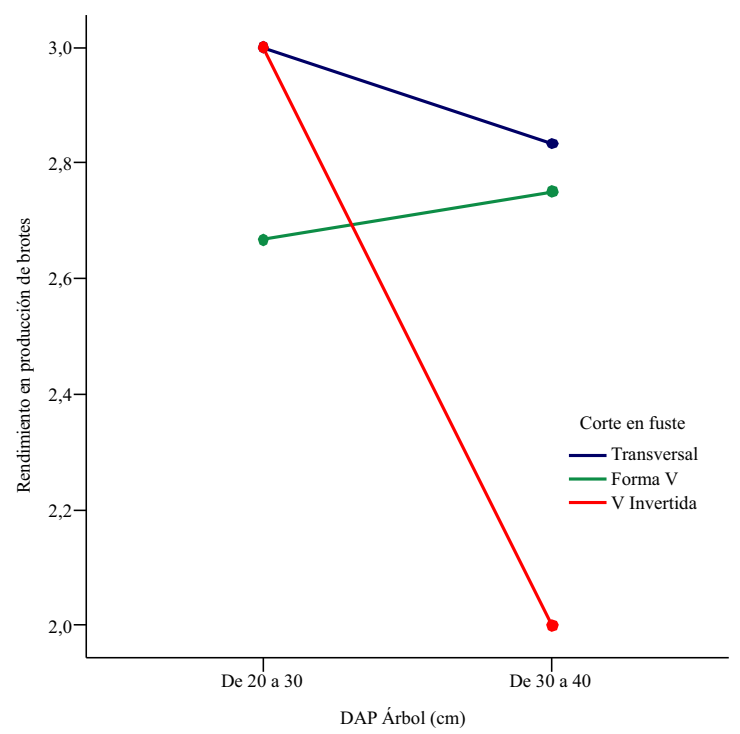

Figura 7. Rendimiento en producción de brotes de ishpingo

\section{Intensidad de selección de Árboles Plus}

Realizada la evaluación de los árboles candidatos sobre los aspectos dasométricas, capacidad de producción de brotes y otros criterios aplicados, se logró la selección de Árboles Plus de 3 especies maderables, tal como se indica en la figura 8.

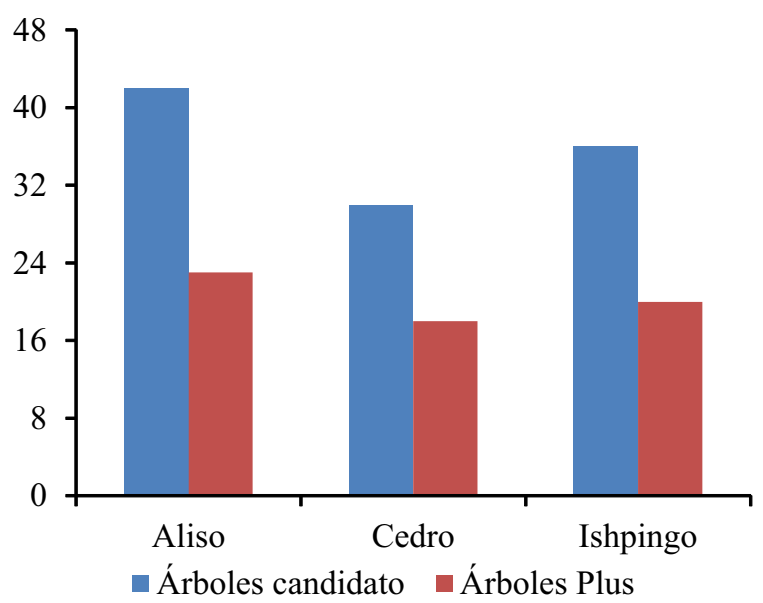

Figura 8. Intensidad de selección de Árboles Plus de 3 especies forestales maderables

En consecuencia el trabajo conllevó a determinar a partir de una población de 108 árboles candidatos se seleccionaron 61 árboles a los que denominamos Árboles Plus, distribuidos en 23 árboles de aliso, 18 de cedro y 23 de ishpingo.

\section{IV.DISCUSIÓN}

Según, Prado (2005) sostiene que, las características que definen la selección de los Árboles Plus se basan principalmente en características de heredabilidad como rectitud de fuste, medida de DAP, árbol libre de plagas y enfermedades y sin presencia de ramas gruesas a nivel de fuste. En el presente trabajo se aplicaron criterios técnicos para la selección de Árboles Plus (DAP, sanidad de árbol, rectitud de fustey otras medidas dasométricas), los mismos que concuerdan con los ya mencionados por el autor, y la aplicación de estos criterios permitieron la selección de los Árboles Plus de las 3 especies forestales estudiadas.

Si bien es cierto que el número de individuos identificados en el período de observación desarrollado no permiten obtener conclusiones definitivas contundentes sobre el comportamiento fenológico de las espe- 
cies forestales, esta entrega revela algunas tendencias sobre las fenofases de estas especies. Sobre esto, Lao (2005) recomienda que en casos extremos una información mensual suministra también la fenología de la comunidad forestal, sostiene además que para el estudio fenológico de los árboles tropicales es recomendable el empleo de una muestra de 10 individuos por especie, seleccionada en orden de aparición en el bosque. Sin embargo, cuando esto no es posible, una muestra de cinco individuos escogidos al azar también es suficiente para este tipo de estudio.

Salazar (2001), indica que para seleccionar árboles sobresalientes de especies forestales maderables, es necesario emplear un método adecuado de selección, la metodología para la selección de Árboles Plus se puede resumir de la siguiente manera: ubicación e identificación de árboles, marcado y codificado de árboles, elaboración de mapa de distribución, manejo silvicultural de árboles candidatos, evaluación de la fenología y selección definitiva de Árboles Plus. Esta metodología de selección fue conducida en el presente trabajo de investigación.

\section{CONCLUSIONES}

En la selección fenotípica a partir de una población de 108 árboles, se obtuvieron 61 Árboles Plus, distribuidos en 23 árboles plus de aliso, 18 árboles de cedro y 20 árboles de ishpingo.

De toda la población de árboles sometidos a evaluación observamos los árboles con DAP menor a $30 \mathrm{~cm}$ y aplicado corte transversal del fuste, mostraron rendimientos ascendentes en la producción de brotes juveniles.

También observamos que el periodo de producción de brotes para la especie aliso fue en promedio 74 días, en cedro 84 días y para ishpingo 93 días, se prevé que estos periodos podrían reducirse con mayor precipitación.

\section{REFERENCIAS BIBLIOGRÁFICAS}

Chang, B. 2007. Selección de especies y manejo de semillas forestales. Turrialba (Costa Rica): CATIE.

Gutiérrez, B., P. Quintero, V. Nieto y O. Murillo. 2003. "Enfoque cooperativo para el mejoramiento genético y la conservación de los recursos forestales en Chile, Colombia y Costa Rica". Investigación agraria. Sistema y recursos forestales 12: 111-122.

Jara, L. F. 1994. Selección y manejo de rodales semilleros. Turrialba (Costa Rica): DFSC CATIE.

Lao, R. 2005. Estudio dendrológico de las especies forestales de la Amazonía Peruana. Lima (Perú): Universidad Nacional Agraria La Molina.

Murillo, O. O., G. Badilla y E. Araya. 2010. "Estrategia de mejoramiento genético para el Programa de Conservación y Mejoramiento Genético de Especies Forestales del ITCR/Fundecor, Costa Rica". Revista Forestal Latinoamericana 16: 275-285.

Prado, M. 2005. Criterios para la selección de árboles plus y control fenológico de cinco especies nativas, en condiciones de bosque seco tropical en Chacocente, Nicaragua. Turrialba (Costa Rica): CATIE.

Salazar, A. 2001. Métodos de colección de especímenes para herbario y muestras de madera de árboles forestales. Lima (Perú): Instituto de Investigaciones Forestales Servicio Forestal y de Caza. Universidad Nacional Agraria La Molina.

Sotelo, M. y J. Weber. 1997. "Priorización de árboles agroforestales en la cuenca amazónica del Perú". Agroforestería en las Américas 4 (4): 12-17. 
